\title{
Análisis de un Sistema de Implante para Osteosintesis de Huesos Largos, Mediante Simulación Computacional
}

\author{
Analysis of an Implant System for Osteosynthesis of Long Bones by Computer Simulation
}

\author{
A. Sirolli*; H. Sanzi* \& G. Elvira*
}

SIROLLI, A.; SANZI, H. \& ELVIRA, G. Análisis de un sistema de implante para osteosíntesis de huesos largos, mediante simulación computacional. Int. J. Morphol., 33(2):594-599, 2015.

RESUMEN: El diseño de implantes para osteosíntesis es una tarea en continuo desarrollo. Las nuevas técnicas quirúrgicas, procesos de fabricación y materiales, en conjunto con el uso de herramientas de cálculo de la ingeniería de última generación, a partir de la Simulación Computacional, permiten obtener implantes más precisos y de calidad superior y dedicados especialmente a cada tipo de fractura. El diseño adecuado y detallado a partir de estas herramientas de ingeniería, es sometido a ensayos mecánicos para verificar y asegurar su eficacia y resistencia estructural. Un estudio utilizando "Técnicas Avanzadas de Análisis", a través de la Simulación Computacional, posibilitaría verificar el grado de integridad de la unión de una placa y sus tornillos, en conjunción con el comportamiento biomecánico del hueso fracturado. En este trabajo se presenta la evaluación estructural, a partir del método de elementos finitos, de una placa de compresión dinámica (DCP), considerando la influencia de diferentes tipos de tornillos y su comportamiento frente a la unión e interacción con el hueso. Esta herramienta de ingeniería utilizada para este fin, ayuda al diseñador a obtener un diseño seguro y adecuado que facilitará el apoyo a cirujanos ortopédicos en la selección de implantes biomecánicos, teniendo en cuenta el tamaño de la fractura y características del hueso.

PALABRAS CLAVE: Bioingeniería; Osteosíntesis; Fractura; Elementos Finitos.

\section{INTRODUCCIÓN}

La función de un dispositivo mecánico para la fijación interna entre huesos que se han fracturado, consiste desde el comienzo del tratamiento, en la inmovilización y rigidización de las partes que garanticen una osteosíntesis estable y permanente, es decir mecánicamente una placa de osteosíntesis debe transmitir las fuerzas desde un extremo al otro del hueso, protegiendo el área de fractura y manteniendo la correcta alineación de los fragmentos durante la reparación.

Muchos son los estudios que se han realizado a través del uso de técnicas avanzadas de cálculo, como es la Simulación Computacional, a partir del método de elementos finitos, para obtener un diseño óptimo y duradero, con resultados satisfactorios en el tiempo (Tovar et al., 2002; Zambrano \& Müller-Karger, 2008; Charles-Harris et al., 2004; Grasa et al., 2008).

El objetivo de este trabajo fue evaluar a partir del método de elementos finitos, de una placa de compresión dinámica (DCP), considerando la influencia de diferentes tipos de tornillos y su comportamiento frente a la unión e interacción con el hueso.

Previamente y con el objeto de convalidar el modelo computacional propuesto, se comparan los resultados obtenidos del ensayo de flexión de cuatro puntos, según IRAM 9426 (Instituto Argentino de Normalización y Certificación, 2014a), utilizado para la certificación de la placa DCP, con los correspondientes obtenidos del modelo de elementos finitos. Esta comparación permitirá validar el modelo propuesto para futuros desarrollos como el que se presenta en este trabajo.

Tipo de fracturas tratadas para su tratamiento biomecánico. La DCP fue introducida en el año 1969, que con un nuevo diseño de agujeros, permitía la compresión axial mediante la inserción de un tornillo excéntrico. La placa puede funcionar de diferentes formas, compresión, neutralización, como tirante o sostén (Rüedi \& Murphy, 2003). En la Figura 1 se presentan dos esquemas de aplicación de una placa sosteniendo un hueso fracturado. 


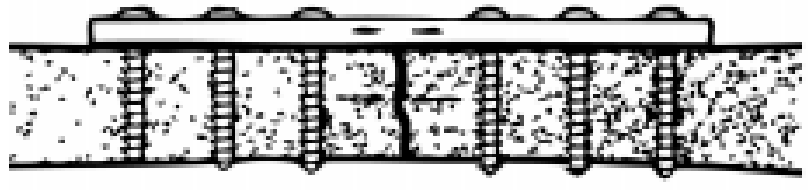

Fig. 1. Aplicación de una placa sosteniendo un hueso fracturado.

En presencia de un defecto óseo, la placa actúa como puente o soporte, fijando los fragmentos manteniéndolos en la alineación adecuada (Fig. 2), es decir, la placa inicialmente soporta completamente la carga que toma el hueso en condiciones normales.

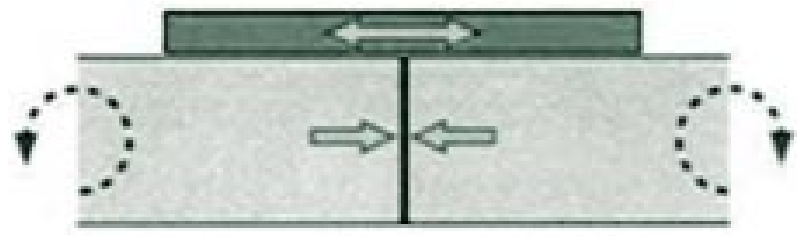

Fig. 2. Esquema de transmisión de fuerzas cuando la placa actúa como tirante.

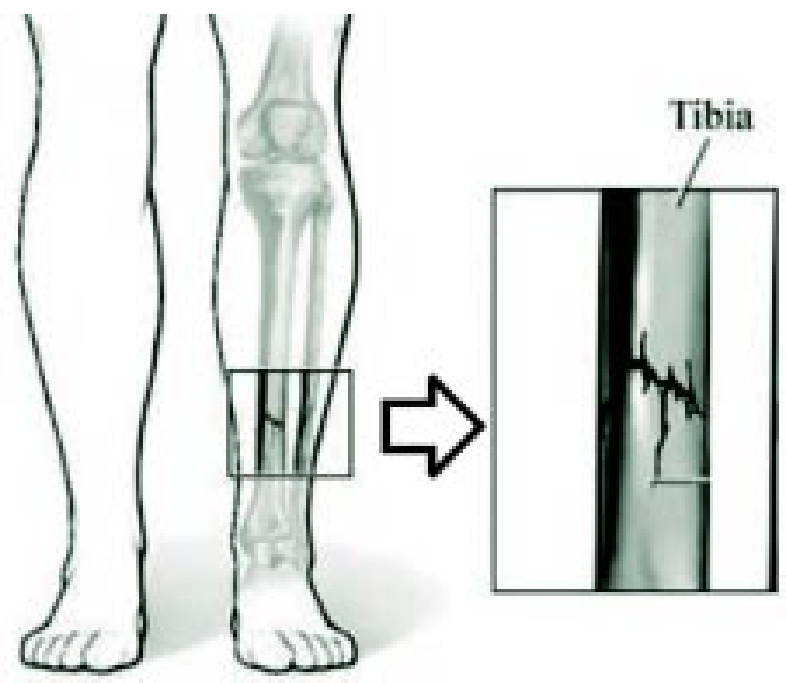

Fig. 3. Esquema del hueso fracturado, tibia.
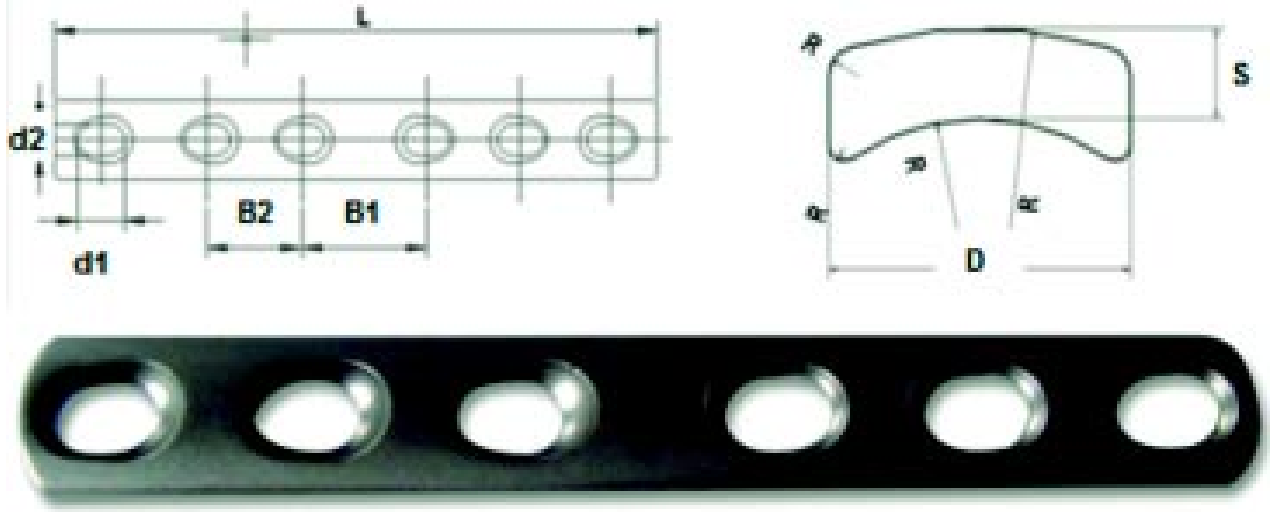

Fig. 4. Placa de Compresión tipo DCP. 


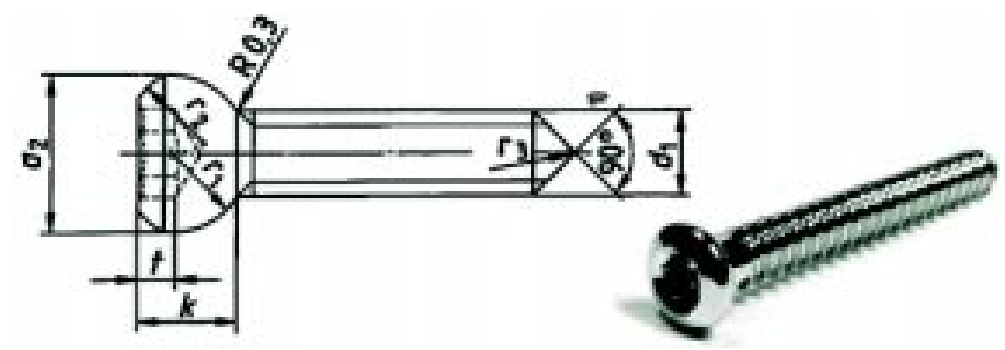

Fig. 5. Detalle perfil del tornillo y su cabeza, según norma IRAM 9423-1 (Instituto Argentino de Normalización y Certificación, 2014c).

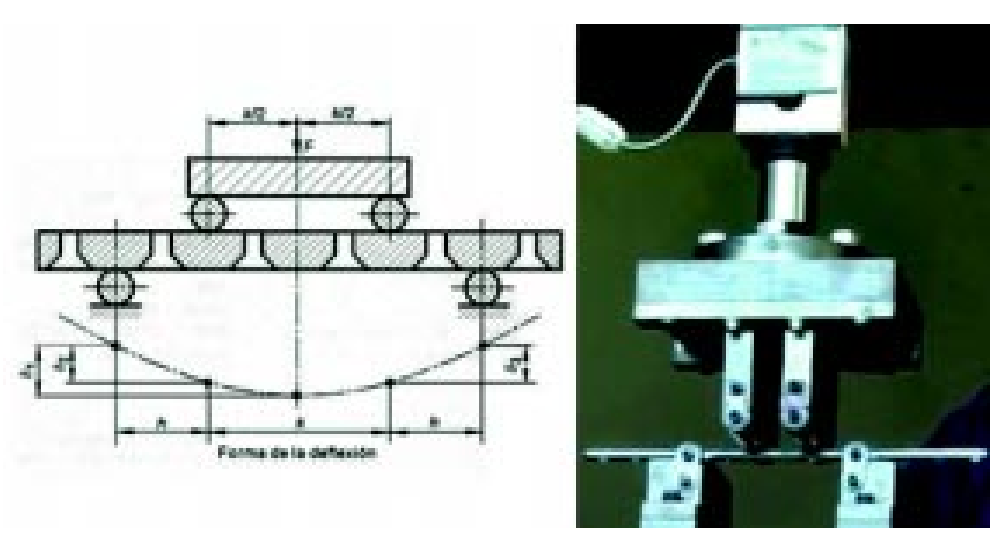

Fig. 6. Disposición general para el ensayo de flexión, según IRAM 9426 (Instituto Argentino de Normalización y Certificación, 2014a).

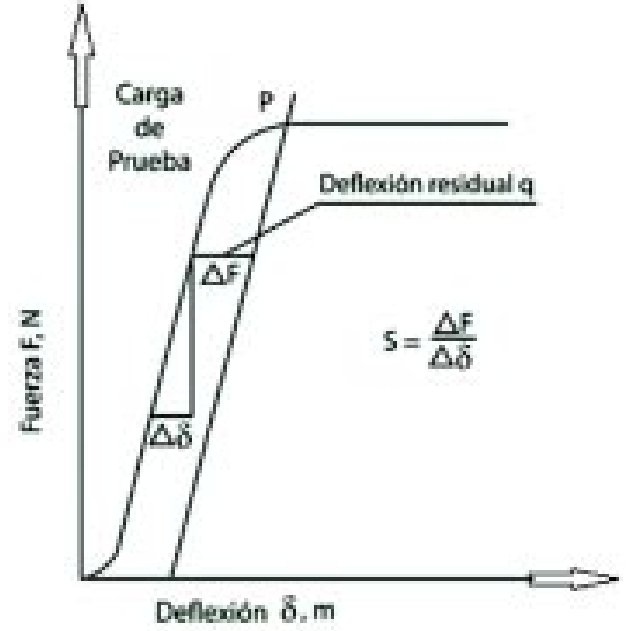

Fig. 7. Curva del ensayo, Carga vs. Desplazamiento.

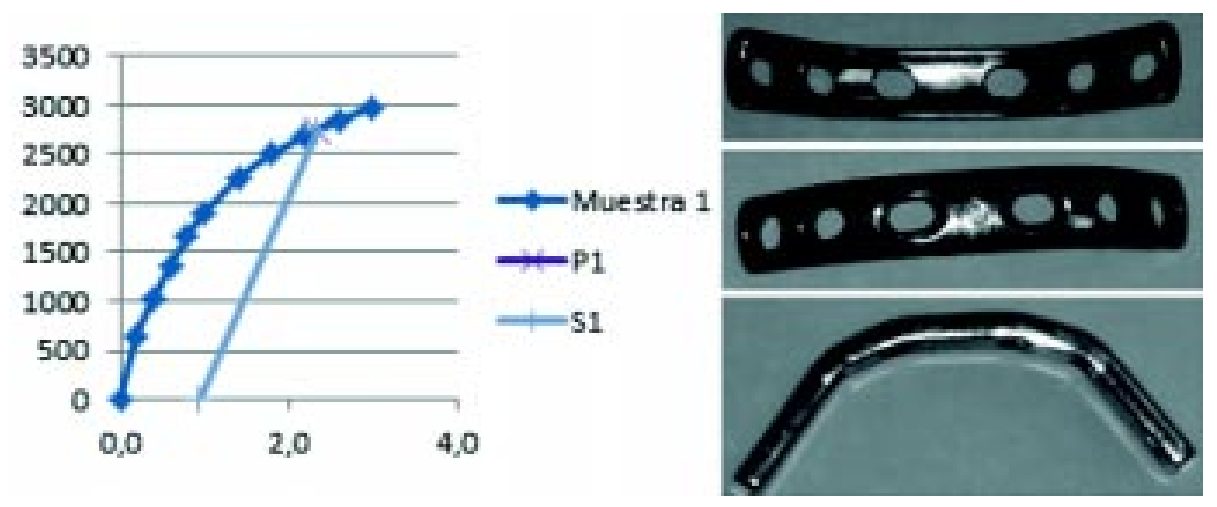

Fig. 8. Resultado del Ensayo Carga (N) en función del desplazamiento (mm).

Para certificar el diseño de la placa DCP se debe cumplir con el siguiente procedimiento de ensayo, que consiste en flexionar dicha placa mediante un dispositivo estructural (Fig. 6). El resultado del ensayo consiste en graficar la curva Carga vs. Desplazamiento (Fig. 7), donde se establece el umbral máximo a partir del cual, es aceptable el comportamiento de la placa en el límite de la plastificación.

Para la placa DCP en estudio (Fig. 4), se han graficado los resultados obtenidos del ensayo y su deformación final (Fig. 8). 


\section{RESULTADOS}

Simulación Computacional del Ensayo. Para convalidar y optimizar el modelo computacional que será utilizado para determinar el comportamiento estructural de la placa de compresión dinámica (DCP), con diferentes tipos de tornillos y su unión al hueso, se presenta un modelo de la placa tridimensional con el objeto de comparar los resultados con los obtenidos del ensayo.

Teniendo en cuenta la simetría geométrica y de cargas transversal, así como longitudinal de la placa, solo se modeló un cuarto de la misma, colocando las condiciones de borde adecuadas y su restricción en los puntos de apoyo correspondientes del dispositivo. La magnitud de la carga aplicada y su ubicación, según el ensayo, coincidente con el inicio de la plastificación, del orden de los $1030 \mathrm{~N}$.

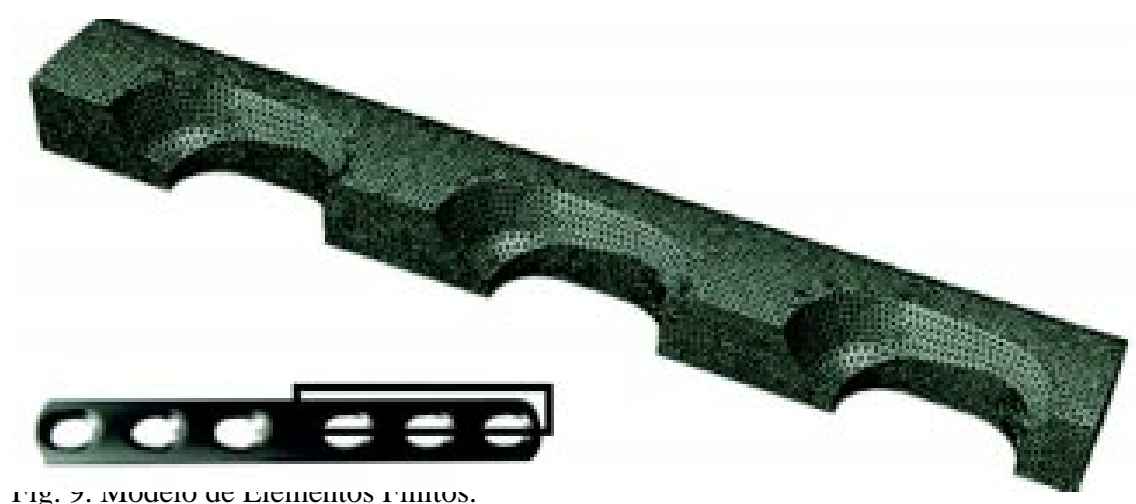

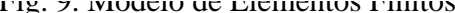

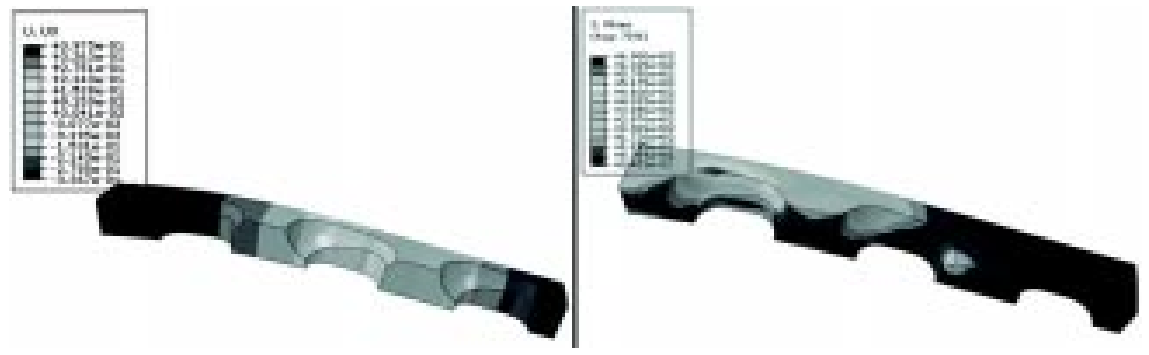

Fig. 10. Tensiones de Von Mises (MPa) y Deformaciones (mm).
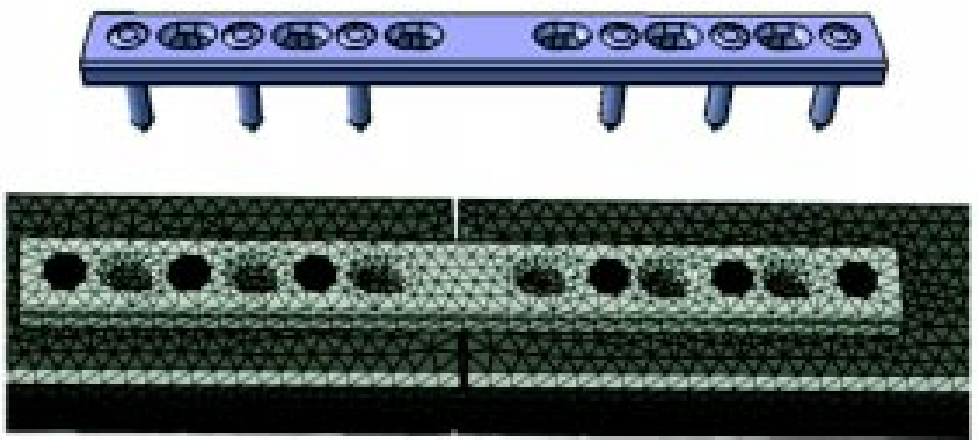

Fig. 11. Modelo de Elementos Finitos del conjunto placa tornillo y hueso.
El modelo quedo conformado por 35742 nodos y 185856 elementos del tipo Sólido Tridimensional del programa ABAQUS 6.9 (2011) (Fig. 9). En la Figura 10, se presentan las tensiones equivalentes de Von Mises y la deformada de la placa, para la carga propuesta.

De los resultados, se aprecia que para la carga aplicada, las tensiones máximas sobre la placa alcanzan valores de plastificación, coincidente con la magnitud de la deformación transversal, obtenidas del ensayo. En función de los resultados obtenidos, el modelo de elementos finitos propuesto será utilizado para futuros desarrollos, como el que se presenta en este trabajo.

\section{Integridad estructural de la union placa-tornillo-hueso}

fracturado. Según las características biomecánicas de la unión, y considerando una fractura de hueso largo (tibia), se propone un modelo tridimensional de los elementos que conforman dicha unión, incluyendo el hueso.

A partir del modelo optimizado, pero para una placa de mayor eficiencia, considerando tres tornillos de fijación a ambos lados de su centro (Fig. 11), se analiza la integridad mecánica estructural de los elementos que conforman la unión.

El modelo fue confeccionado utilizando elementos del tipo Sólido del Programa ABAQUS, con una formulación de segundo orden y altamente densificado el cual quedó conformado por 335000 elementos. En la Figura 12, se presenta en forma separada, el estado tensional de Von Mises para las placas y los tornillos de fijación. Se observa que las máximas tensiones se alcanzan en la cabeza del tornillo, en su interior. Se aprecia diferencias notables en el nivel de tensiones, cuando se modifica la profundidad de ajuste. 


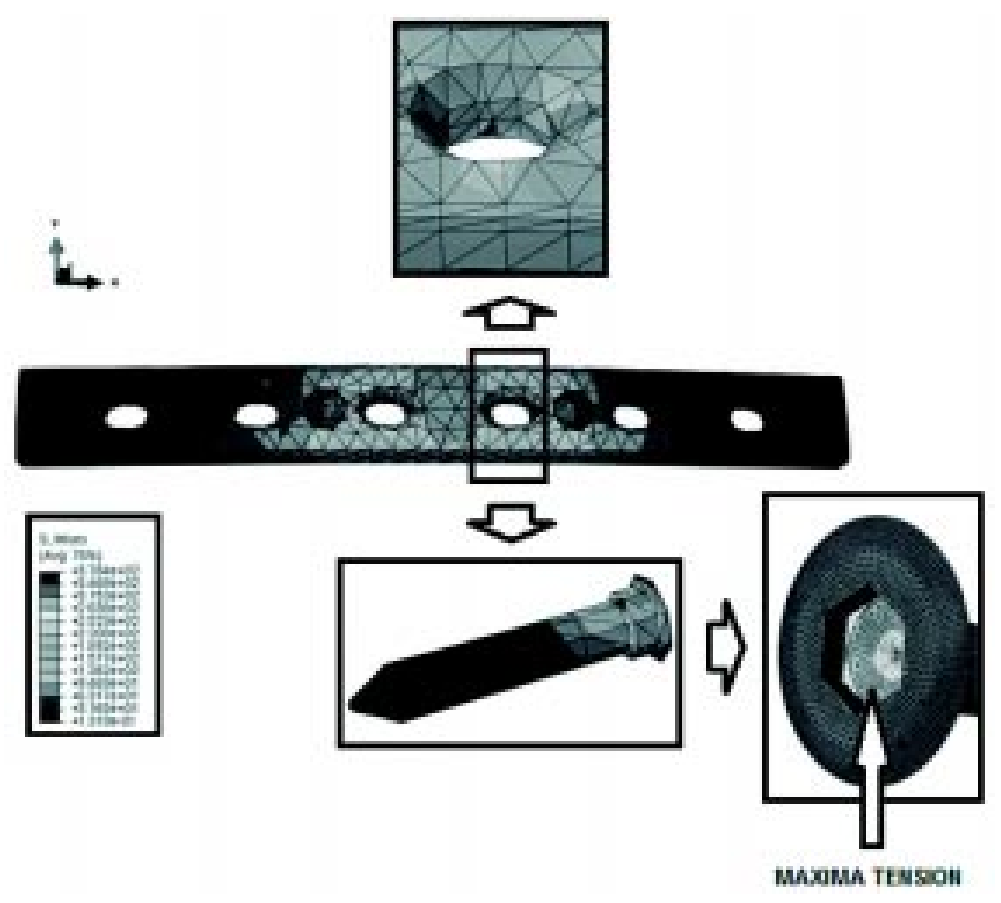

Fig. 12. Tensiones de Von Misses (MPa) en la placa y tornillos.
La profundidad del orificio de ajuste, es vital importancia para asegurar el comportamiento adecuado del tornillo. Una profundidad excesiva puede provocar la fractura durante el ajuste final o torque del tornillo sobre la placa. Este es un estudio para encarar en futuros desarrollos.

Cabe destacar que una cabeza hexagonal profunda permite un buen alojamiento de la herramienta de apriete (destornillador), facilitando una buena operación del asentamiento y ajuste final, pero como desventaja, dicha medida o profundidad debilita el tornillo, con la posibilidad que durante el ajuste, en el momento de la colocación del implante, se produzca su rotura.

Las normas de aplicación solo establecen un valor mínimo de profundidad pero no el máximo. Por lo tanto, resulta fundamental determinar dicho límite para evitar la fractura.

\section{DISCUSIÓN}

Previamente y con el objeto de convalidar un modelo computacional completo y detallado, incorporando el hueso, como se presentó en esta última parte, se compararon los resultados obtenidos del ensayo de flexión de cuatro puntos, según IRAM 9426 (Instituto Argentino de Normalización y Certificación, 2014a), utilizado para la certificación de la placa DCP, con los correspondientes obtenidos del modelo de elementos finitos.

A partir de esta referencia, se planteó un nuevo modelo tridimensional de elementos finitos, incorporando la placa de mayor longitud y cantidad de tornillos, incluyendo el hueso, con el objeto de determinar la integridad de los elementos estructurales portantes.

De los resultados obtenidos, se observa que la placa en la zona de los agujeros es la más comprometida desde el punto de vista tensional, particularmente para los agujeros centrales. Los tonillos, en la zona por debajo de la cabeza, donde ajusta con la placa mediante la rosca de bloqueo, es la más comprometida.

Como se observó en los resultados, las máximas tensiones se alcanzan en la cabeza del tornillo. Se aprecia diferencias notables en el nivel de tensiones, cuando se incrementa la profundidad de ajuste.
Por otro lado, es probable que una placa de mayor longitud y con mayor cantidad de tornillos, presente un comportamiento estructural y biomecánico más eficiente.

Queda para futuros desarrollos la posibilidad de estudiar el comportamiento estructural y biomecánico, con presencia del hueso, para placas de diferente tamaño, ya sea en longitud y espesor, con tornillos de ajuste que aseguren un comportamiento óptimo en el momento de llevar a cabo el apriete final en el quirófano.

Un estudio como el presentado, utilizando "Técnicas Avanzadas de Análisis", a través de la Simulación Computacional, ha permitido verificar el grado de integridad de la unión de la placa, tornillos, en conjunción con el comportamiento biomecánico con el hueso fracturado.

Esta herramienta de ingeniería utilizada para este fin, ayuda al diseñador a obtener un diseño seguro y adecuado que facilitará el apoyo a cirujanos ortopédicos en la selección de implantes biomecánicos, teniendo en cuenta el tamaño de la fractura y características del hueso.

AGRADECIMIENTOS. Agradecemos al INSTITUTO NACIONAL DE TECNOLOGIA INDUSTRIAL (Argentina - Córdoba) por su colaboración en esta investigación permitiéndonos el uso del programa Abaqus. 
SIROLLI, A.; SANZI, H. \& ELVIRA, G. Analysis of an implant system for osteosynthesis of long bones by computer simulation. Int. J. Morphol., 33(2):594-599, 2015.

SUMMARY: The design of implants for osteosynthesis is a work in continuous development. New surgical techniques, manufacturing processes and materials, together with the use of tools for calculating the art engineering, from Computational Simulation allow more precise and top quality implants especially designed for each type of fracture. The adequate and detailed design based on these engineering design tools, is subjected to mechanical testing to verify and ensure their effectiveness and structural strength. A study using "Advanced Technical Analysis" through Computational Simulation, makes it possible to verify the degree of integrity of the union of a plate and screws in conjunction with the biomechanical behavior of the fractured bone. This paper presents structural assessment, from the finite element method, a dynamic compression plate (DCP), considering the influence of different types of screws and their behavior in binding and interaction with the bone. This engineering tool used for this purpose, helps the designer to ensure safe and proper designs that facilitate support for orthopedic surgeons in selecting biomechanical implants, considering the size and characteristics of the bone fracture.

KEY WORDS: Bioengineering; Osteosynthesis; Fracture; Finite Element.

\section{REFERENCIAS BIBLIOGRÁFICAS}

ABAQUS 6.9. User Documentation - Internet Manual. Simulia, 2011. Disponible en: http://abaqusdoc.ucalgary.ca/v6.9/books/ usi/default.htm

Charles-Harris, M; Lacroix, D.; Proubasta, I. \& Planell, J. A. Clavos intramedulares vs. placas de osteosíntesis para fracturas de fémur: Análisis por elementos finitos. Biomec., 12(1):6470,2004

Grasa, J.; González, L. A.; Gómez Benito, M. J.; García-Aznar, J. M.; Bea, J. A.; Laborda, P. J. \& Doblaré, M. Caracterización mecánica de las propiedades del tejido óseo cortical. An. Mec. Fract., 25(1):23-8, 2008.

Müller, M. E.; Nazarian, S. \& Koch, P. Classification AO des fractures. Tome I. Les os longs. Berlin, Springer-Verlag, 1987.

Instituto Argentino de Normalización y Certificación (IRAM). Norma IRAM 9426:1999 Implantes quirúrgicos. Placas para osteosíntesis. Determinación de la rigidez y de la resistencia a la flexión. Buenos Aires, Instituto Argentino de Normalización y Certificación (IRAM), 2014a. Disponible en: http:// aplicaciones.iram.org.ar/carritoiram/BuscainicialCD.asp

Instituto Argentino de Normalización y Certificación (IRAM). Norma IRAM 9401-2:2005. Implantes Quirúrgicos, Materiales Metálicos, Acero Inoxidable Austenítico Semielaborado de bajo contenido de carbono. Buenos Aires, Instituto Argentino de Normalización y Certificación (IRAM), 2014b. Disponible en: http://aplicaciones.iram.org.ar/carritoiram/ BuscainicialCD.asp

Instituto Argentino de Normalización y Certificación (IRAM). Norma IRAM 9423-1:2005. Implantes quirúrgicos. Tornillos metálicos para hueso. Parte 1: Con hexágono embutido y rosca asimétrica tipos HA y HB. Medidas y tolerancias. Buenos Aires, Instituto Argentino de Normalización y Certificación
(IRAM), 2014c. Disponible en: http://aplicaciones.iram.org.ar/ carritoiram/BuscainicialCD.asp

Rüedi, T. P. \& Murphy, W. M. Principios de la AO en el tratamiento de las fracturas. Barcelona, Masson, 2003.

Tovar, C.; Cerrolaza, M. \& Bendayán, J. Diseño y análisis por elementos finitos de placas para fijación interna de fracturas. En: Goicolea, M.; Mota Soares, C.; Pastor, M. \& Bugeda, G. (Eds.). Métodos Numéricos en Ingeniería V. Barcelona, Semni, 2002.

Zambrano, L. A. \& Müller-Karger, C. Estudio del efecto de placas de fijación en fracturas de tibia proximal utilizando el método de elementos finitos. Bol. Tec. I. M. M. E., 46(3):43-60, 2008.

\author{
Dirección para Correspondencia: \\ Agustin Sirolli \\ Grupo Investigación Ingeniería Estructural GIIE \\ Universidad Tecnológica \\ Nacional Facultad Regional Haedo \\ París 532 Haedo \\ Buenos Aires \\ ARGENTINA
}

Email: agustinsirolli@yahoo.com.ar

Recibido : 09-12-2014

Aceptado: 14-04-2015 